\title{
Characterizations of variational convergence of bifunctions defined on products of two sets
}

\author{
Huynh Thi Hong Diem*
}

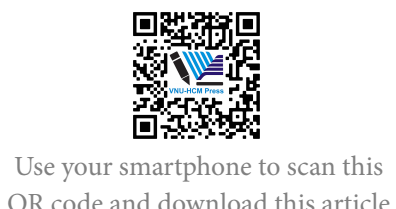

QR code and download this article
Department of Mathematics, Ho Chi Minh City University of Technology, VNU-HCM, Vietnam

\section{Correspondence}

Huynh Thi Hong Diem, Department of Mathematics, Ho Chi Minh City University of Technology, VNU-HCM, Vietnam

Email: hthdiem@hcmut.edu.vn

History

- Received: 9-12-2019

- Accepted: 20-11-2020

- Published: 31-12-2020

DOI : 10.32508/stdjet.v3iSI3.643

\section{Check for updates}

\section{Copyright}

(c) VNU-HCM Press. This is an openaccess article distributed under the terms of the Creative Commons Attribution 4.0 International license.

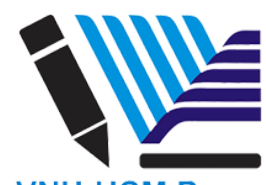

VNU-HCM Press

\begin{abstract}
We present definitions of types of variational convergence of finite-valued bifunctions defined on rectangular domains and establish characterizations of these convergences. In the introduction, we present the origins of the research on variational convergence and then we lead to the specific problem of this paper. The content of the paper consists of 3 parts: variational convergance of fucntion; variational convergance of bifunction; and characterizations of variational convergence of bifunction, this part is the main results of this paper. In section 2, we presented the definition of epi convergence and presented a basic property problem that will be used to extend and develop the next two sections. In section 3, we start to present a new definition, the definition of convergence epi / hypo, minsup and maxinf. To clearly understand of these new definitions we have provided comments (remarks) and some examples which reader can check these definitions. The above contents serve the main result of this paper will apply in part 4. Now, we will explain more detail for this part as follows. Firstly, variational convergence of bifunctions is characterized by the epi- and hypo-convergence of related unifunctions, which are slices sup- and inf-projections.

The second characterization expresses the equivalence of variational convergence of bifunctions and the same convergence of the so-called proper bifunctions defined on the whole product spaces. In the third one, the geometric reformulation, we establish explicitly the interval of all the limits by computing formulae of the left- and right-end limit bifunctions, and this is necessary and sufficient conditions of the sequence bifunctions to attain epi / hypo, minsup and maxinf convergence.
\end{abstract}

Key words: Variation convergence, Epi-convergence, Hypo-convergence, Epi/hypo-convergence, Lopsided convergence, Equivalence class

\section{INTRODUCTION}

Epi-convergence, epi/hypo-convergence, and lopsided convergence are the main types of variational convergence, of unifunctions (briefly, functions) and bifunctions, respectively. Epi-convergence was introduced independently ${ }^{1-3}$. For details of this convergence and its applications, the reader is referred to the books ${ }^{4-6}$.

Epi/hypo-convergence of extended-real-valued bifunctions was proposed and developed ${ }^{7,8}$. Typical references for this convergence and its applications $^{9-12}$. After the appearance ${ }^{7}$, lopsided convergence, a modified verson stronger than epi/hypoconvergence was further studied ${ }^{13}$. All the aforementioned contributions investigate the class of extendedreal-valued functions defined on the whole space $X$, denoted by $f \mathrm{cn}(X)$, or of bifunctions defined on the whole product space $X \times Y$ denoted by biv $(X \times Y)$. Lopsided convergence was discussed for the first time for a class of finite-valued bifunctions defined on $A \times$ $B$, where $A \subset X$ and $B \subset Y$ with $X, Y$ being finitedimensional spaces ${ }^{7}$.
This class is also called finite-valued bifunctions on rectangles and designated by fv-biv $(X \times Y)$. It is important because typical bifunctions in many mathematical models of practical problems are Lagrange functions ${ }^{14,15}$ in constrained optimization, Hamilton functions in variational calculus and optimal control, and payoff functions in zero-sum games, all belong to this class. Epi/hypo-convergence of finite-valued bifunctions on rectangles was studied ${ }^{16,17}$. Lopsided convergence for special subclasses of fv-biv $(X \times Y)$ was investigated $^{18-20}$.

Lopsided convergence is stronger than epi/hypoconvergence. Hence, it has more beautiful properties. But, it is not symmetric with respect to the two components $x \in X$ and $y \in Y$. It has "one-sided" properties and is appropriated for either minsup- or maxinfproperties. However, the first variational properties of bifunctions attracting attention from researchers must be saddle-point properties ${ }^{21-23}$, which combine both the minsup- and maxinf-properties. Especially, such properties are crucial for studies of dualities in general and dual problems ${ }^{24}$ in particular. Observe 
further that recently lopsided convergence of finitevalued bifunctions and its applications were intensively studied for special types of bifunctions with applications mainly in special important classes of problems. However, for epi/hypo-convergence of finitevalued bifunctions, we see only papers ${ }^{16,17}$ for the finite-dimensional case.

Motivated by the above brief about results on variational convergence obtained up to this time, in this paper we study variational convergence of finitevalued bifunctions on rectangles in the case of general metric spaces. Our aim is characterizations of such a convergence. The obtained results are new in various aspects or improve/extend the corresponding ones ${ }^{16-18,25,26}$ for the finite-dimensional case to the general metric-space case.

Namely, we develop three characterizations of the epi/hypo- and lop-convergence of bifunctions in $\mathrm{fv}$ biv in the general metric-space case. It should be stressed that characterizations of variational convergence are important not only for theoretical developments but in fact even more crucial for applications. In this paper, all the spaces are metric spaces, if not otherwise specified. Our notation is standard. For instance, $\mathbb{R}, \overline{\mathbb{R}}$ and $\mathbb{N}$ stand for the real line, the extended real line $\overline{\mathbb{R}}:=\mathbb{R} \cup\{-\infty,+\infty\}$, and the set of the natural numbers, respectively (resp). For $A \subset X$, int $A$ and bd $A$ denote the interior and the boundary of $A$, resp. Function $\varphi: X \rightarrow \overline{\mathbb{R}}$ is called lower semincontinuous (lsc, upper semincontinuous (usc)) at $\bar{x}$ if $\liminf _{x \rightarrow \bar{x}} \varphi(x) \geq \varphi(\bar{x})\left(\limsup _{x \rightarrow \bar{x}} \varphi(x) \leq\right.$ $\varphi(\bar{x})$, resp). For $A^{k} \subset X$, the lower/inner limit and the upper/outer limit are defined by

$\operatorname{Liminf}_{k} A^{k}:=\left\{x \in X: \lim _{k} x^{k}=x\right.$, for $\left.x^{k} \in A^{k}\right\}$,

$\operatorname{Limsup}_{k} A^{k}:=\left\{x \in X: \lim _{l} x^{k_{l}}=x\right.$, for $\left.x^{k_{l}} \in A^{k_{l}}\right\}$,

$x^{k_{l}}$ being subsequence of $\left.x^{k}\right\}$.

If $\operatorname{Liminf}_{k} A^{k}=\operatorname{Limsup}_{k} A^{k}$, we say that $A^{k}$ tend to $A$ in the Painlevé-Kuratowski sense, denoted by $A^{k} \stackrel{P-K}{\longrightarrow}$ $A$ or $A=\operatorname{Lim}_{k} A^{k}$. Later on, we also write simply li, ls, Li, Ls instead of liminf, limsup, Liminf, Limsup, resp.

\section{VARIATIONAL CONVERGENCE OF FUNCTIONS}

In this section, we present epi-convergence of functions and its basic variational properties. Let $X$ be a metric space, $A^{k}, A \subset X$ be nonempty sets, and $\left\{\varphi^{k}: A^{k} \rightarrow \mathbb{R}\right\}_{k}, \varphi: A \rightarrow \mathbb{R}$ be elements of $\mathrm{fv}$-fon $(X)$. The following definition extends the corresponding notion ${ }^{26}$ from the case of finite-dimensional spaces to that of general metric spaces.

Definition 1 (epi-convergence) $\left\{\varphi^{k}\right\}_{k}$ in fv-fon $(X)$ is said to epi-converge to $\varphi \mathrm{fv}$-fon $(X)$, denoted by $\varphi=e-\lim _{k} \varphi^{k}$ or $\varphi^{k} \stackrel{e}{\rightarrow} \varphi$, if (a) for all $x^{k} \in A^{k} \rightarrow x, \operatorname{li}_{k} \varphi^{\mathrm{k}}\left(\mathrm{x}^{\mathrm{k}}\right) \geq \varphi(\mathrm{x})$ when $x \in A$ and $\varphi^{k}\left(x^{k}\right) \rightarrow+\infty$ when $x \notin A$;

(b) for all $x \in A$, there exist $x^{k} \in A^{k} \rightarrow x$ such that $\operatorname{ls}_{k} \varphi^{k}\left(x^{k}\right) \leq \varphi(x)$.

Definition 2 (lower and upper epi-limits) Let $\varphi^{k} \in$ $\mathrm{fv}$-fon $(X)$ and $x \in X$. The lower epi-limit of $\left\{\varphi^{k}\right\}_{k}$ at $x$ is $\operatorname{eli} \varphi^{k}(x):=\inf _{\left\{x^{k} \in A^{k} \rightarrow x\right\}} \operatorname{li}_{k} \varphi^{k}\left(x^{k}\right)$, where $\left\{x^{k} \in A^{k} \rightarrow x\right\}$ means that we consider all the possible sequences $x^{k} \in A^{k}$ tending to $\mathrm{x}$.

The upper epi-limit of $\left\{\varphi^{k}\right\}_{k}$ at $x$ is $\operatorname{eli} \varphi^{k}(x):=$ $\inf _{\left\{x^{k} \in A^{k} \rightarrow x\right\}} \operatorname{li}_{k} \varphi^{k}\left(x^{k}\right)$,

It can be proved that $\varphi^{k} \stackrel{e}{\rightarrow} \varphi$ if and only if $\operatorname{eli} \varphi^{k}(x)=$ $\operatorname{els} \varphi^{k}(x)=\varphi(x)$ when $x \in A$ and $\operatorname{eli}^{k}(x)=+\infty$ when $x \notin A$.

The notion symmetric to epi-convergence is hypoconvergence which is defined as follows. $\varphi^{k}$ are called hypo convergent to $\varphi$, designated by $\varphi=h-\lim _{k} \varphi^{k}$ or $\varphi^{k} \stackrel{h}{\rightarrow} \varphi$, if $-\varphi^{k}$ epi-converge to $-\varphi$. Hence, the lower and upper hypo-limits of $\left\{\varphi^{k}\right\}_{k}$ at $x \in A$ are, resp,

$\operatorname{hli} \varphi^{k}(x):=\sup _{\left\{x^{k} \in A^{k} \rightarrow x\right\}} \operatorname{li}_{k} \varphi^{k}\left(x^{k}\right)$, $\operatorname{hl} s \varphi^{k}(x):=\sup _{\left\{x^{k} \in A^{k} \rightarrow x\right\}} l s_{k} \varphi^{k}\left(x^{k}\right)$.

Similar to epi-convergence, $\varphi^{k} \stackrel{h}{\rightarrow} \varphi$ if and only if $\operatorname{hli} \varphi^{k}(x)=\operatorname{hls} \varphi^{k}(x)=\varphi(x)$ when $x \in A$ and $\operatorname{hli} \varphi^{k}(x)=-\infty$ when $x \notin A$.

Now we extend to the metric-space case the basic variational property of epi-convergence, proved $^{14}$ for the finite-dimensional case.

Theorem 1 (basic variational property of epiconvergence $^{6}$ ) For $\varphi^{k}, \varphi \in \mathrm{fv}-\mathrm{fcn}(X)$ with $\varphi=\mathrm{e}-\lim \varphi^{k}$, one has $l s_{k}\left(\inf _{A^{k}} \varphi^{k}(x)\right) \leq \inf _{A} \varphi(x)$. Moreover, if $x^{k_{j}}$ is a minimizer of $\varphi^{k_{j}}$ for all $j \in N$ and $x^{k_{j}} \rightarrow \bar{x} \in A$, then $\bar{x}$ in a minimizer of $\varphi$ and the infimal value of $\varphi^{k_{j}}$ also converge to the infimal value of $\varphi$.

The proof is similar to the known proof for the finitedimensional case and so omitted. The second part can be reformulated equivalently as follows: if $\mathrm{e}-$ $\lim _{k} \varphi^{k}=\varphi$, then

$A \cap \operatorname{Ls}_{\mathrm{k}}\left(\operatorname{argmin}_{A^{k}} \varphi^{k}\right) \subset \operatorname{argmin}_{A}(\varphi)$.

one can prove easily an extension of this inclusion: if $\varepsilon^{k} \searrow 0$, then

$A \cap \operatorname{Ls}_{\mathrm{k}}\left(\varepsilon^{k}-\operatorname{argmin}_{A^{k}} \varphi^{k}\right) \subset \operatorname{argmin}(\varphi)$.

To have the reverse inclusion with the full Lim replacing Limsup together with the convergence of the corresponding infimal values, one needs the following tightness condition.

Definition 3 (tight epi-convergence) one says that $\left\{\varphi^{k}\right\}_{k}$ epi-converge tightly to $\varphi$ in $\mathrm{fv}$-fon $(X)$ if they epi-converge and for each $\varepsilon>0$, there exists a compact set $B_{\varepsilon}$ in $X$ and $k_{\varepsilon} \in \mathbb{N}$ such that, for all $k \geq$ $k_{\varepsilon}, \inf _{B_{\varepsilon} \cap A^{k}} \varphi^{k} \leq \inf _{A^{k}} \varphi^{k}+\varepsilon$. 
Theorem 2 (basic property of tight epi-convergence ${ }^{6}$ ) (i) If $\varphi^{k}$ tightly epi-converge to $\varphi$ and $\inf _{A^{k}} \varphi$ is finite, then $\inf _{A^{k}} \varphi^{k} \rightarrow \inf _{A} \varphi$. The converge holds if $X$ is finite-dimensional.

(ii) Assume that $\varphi^{k}$ epi-converge to $\varphi$ and $\inf _{A} \varphi$ is finite. If there exist $\varepsilon^{k} \searrow 0$ such that $A \cap$ $\operatorname{Lim}_{\mathrm{k}} \varepsilon^{k}-\operatorname{argmin}_{A^{k}} \varphi^{k}=\operatorname{argmin}_{A} \varphi$, then $\varphi^{k}$ tightly epi-converge to $\varphi$. The converse is true if $\mathrm{X}$ is separable.

To prove this statement, modify suitably the arguments for the finite-dimentional case ${ }^{26}$ and apply the theorem on level sets associated with epiconvergence $^{27}$. Due to the requirement of a limited length of the paper, we skip the details.

\section{VARIATIONAL CONVERGENCE OF BIFUNCTIONS}

For $A, A^{k} \subset X, B, B^{k} \subset Y, \phi^{k}: A^{k} \times B^{k} \rightarrow \overline{\mathbb{R}}$, and $\phi$ : $A \times B \rightarrow$

$\mathrm{R}$, weproposethefollowingdefinition.

Definition 4 (epi/hypo-convergence) Bifunctions $\phi^{k}$ in fv-biv $(X \times Y)$ are called epi/hypo-convergent (e/hconvergent) to $\phi \in \mathrm{fv}$-biv $(X \times Y)$ if

(a) for all $y \in B$ and $x^{k} \in A^{k} \rightarrow x$, there exist $y^{k} \in B^{k} \rightarrow$ $y$ such that $l i_{k} \phi^{k}\left(x^{k}, y^{k}\right) \geq \phi(x, y)$ when $x \in A$ and $\phi^{k}\left(x^{k}, y^{k}\right) \rightarrow+\infty$ when $x \notin A$;

(b) for all $x \in A$ and $y^{k} \in B^{k} \rightarrow y$, there exist $x^{k} \in$ $A^{k} \rightarrow x$ such that $l s_{k} \phi^{k}\left(x^{k}, y^{k}\right) \leq \phi(x, y)$ if $y \in B$ and $\phi^{k}\left(x^{k}, y^{k}\right) \rightarrow-\infty$ when $y \notin B$;

This convergence is denoted by $\phi=e / h-$ $\lim _{k} \phi^{k}$ or $\phi^{k} \stackrel{e / h}{\longrightarrow} \phi$.

Note that if $\varphi^{k}$ do not depend on $y$, then epi/hypoconvergence becomes epi-convergence, and if they do not depend on $\mathrm{x}$, it collapses to hypo-convergence. However, the epi/hypo-convergence of $\phi(\cdot, \cdot)$ is not the epi-convergence of $\phi(x, \cdot)$ to $\phi(\cdot, y)$ for all $y$ together with the hypo-convergence of $\phi^{k}(x, \cdot)$ to $\phi(x, \cdot)$ for all $x$. This is a sufficient condition for $\mathrm{e} / \mathrm{h}$ convergence, but not a necessary condition. We see that $\mathrm{e} / \mathrm{h}$-convergence and h/e-convergence are symmetric. Moreover, h/e-convergence coincides with e/h-convergence, if one keeps taking minimum on $\mathrm{x}$ and maximum on $y$, only changes the order of the two operations. The lopsided convergence defined below is our extension to the metric-space case of the corresponding concept for the finite-dimensional case ${ }^{26}$.

Definition 5 (minsup-lop convergence)

$\phi^{k} \in \mathrm{fv}-$ biv $(X \times Y)$ are called minsup-lopsided (minsup-lop) convergent to $\phi \in \mathrm{fv}-$ biv $(X \times Y)$ if (a) for each $y \in B$ and $x^{k} \in A^{k} \rightarrow x$, there exist $y^{k} \in$ $B^{k} \rightarrow y$ such that $l i_{k} \phi^{k}\left(x^{k}, y^{k}\right) \geq \phi(x, y)$ when $x \in A$ and $\phi^{k}\left(x^{k}, y^{k}\right) \rightarrow+\infty$ when $x \notin A$; (b) for all $x \in A$, there exist $x^{k} \in A^{k} \rightarrow x$ such that, for all $y^{k} \in B^{k} \rightarrow y, l s_{k} \phi^{k}\left(x^{k}, y^{k}\right) \leq \phi(x, y)$ when $y \in B$ and $\phi^{k}\left(x^{k}, y^{k}\right) \rightarrow-\infty$ when $y \notin B$.

This convergence is denoted by $\phi=\operatorname{mins}-$ $\lim _{k} \phi^{k}$ or $\phi^{k} \stackrel{\operatorname{mins}}{\longrightarrow} \phi$.

Clearly the roles of $x$ and $y$ are not symmetric in this definition. Changing the order of $x$ and $y$ leads to the following definition of maxinf-lop convergence:

(a) for all $x \in A$ and $y^{k} \in B^{k} \rightarrow y$, there exist $x^{k} \in$ $A^{k} \rightarrow x$ such that $l s_{k} \phi^{k}\left(x^{k}, y^{k}\right) \leq \phi(x, y)$ if $y \in B$ and $\phi^{k}\left(x^{k}, y^{k}\right) \rightarrow-\infty$ ify $\notin B$;

(b) for all $y \in B$ and $x^{k} \in A^{k} \rightarrow x$, there exist $y^{k} \in B^{k} \rightarrow$ $y$ such that, for all $x^{k} \in A^{k} \rightarrow x, l i_{k} \phi^{k}\left(x^{k}, y^{k}\right) \geq \phi(x, y)$ if $x \in A$ and $\phi^{k}\left(x^{k}, y^{k}\right) \rightarrow+\infty$ if $x \notin A$.

We denote this convergence by $\phi=\operatorname{maxi}-$ $\lim _{k} \phi^{k}$, or $\phi^{k} \stackrel{\text { maxi }}{\longrightarrow} \phi$.

It is obvious that each minsup-lop or maxinf-lop convergence implies e/h-convergence. Condition (a) of e/h-convergence and minsup-lop convergence are the same. While condition (b) of lop-convergence is stronger (b) of e/h-convergence. Indeed, for $x \in A$ lop-convergence requires the existence of a common sequence $x^{k} \in A^{k} \rightarrow x$ such that for all $y^{k} \in B^{k} \rightarrow y$, $l s_{k} \phi^{k}\left(x^{k}, y^{k}\right) \leq \phi(x, y)$ if $y \in B$ and $\phi^{k}\left(x^{k}, y^{k}\right) \rightarrow$ $-\infty$ if $y \notin B$. Surely, this $\left\{x^{k}\right\}_{k}$ satisfies (b) of e/hconvergence. However, conversely, if one has $\phi^{k} \stackrel{e / h}{\longrightarrow}$ $\phi$, one still cannot derive that $\phi^{k}$ minsup-lop converge to $\phi$ as shown by the following example.

Example 1 Let $A^{k}=B^{k}=[1 / k, 1], A=B=[0,1]$, and

$$
\phi^{k}(x, y)=\left\{\begin{array}{l}
1 \text { if }(x, y) \in A^{k} \times B^{k}, x \neq y, \\
0 \text { if }(x, y) \in A^{k} \times B^{k}, x=y .
\end{array}\right.
$$

Then,

$$
\phi(x, y)=\left\{\begin{array}{l}
1 \text { if }(x, y) \in[0,1]^{2}, x \neq y \\
0 \text { if }(x, y) \in[0,1]^{2}, x=y
\end{array}\right.
$$

Clearly $\phi^{k} \stackrel{e / h}{\longrightarrow} \phi$. We show that condition (b) of minsup-lop convergence is violated. For $x=0$ and any $x^{k} \in A^{k} \rightarrow x$, we take $y=0$ and $y^{k} \in B^{k} \rightarrow 0$ such that $y^{k} \neq x^{k}$ for all $k$. Then, $l s_{k} \phi^{k}\left(x^{k}, y^{k}\right)=1>0=$ $\phi(x, y)$.

Remark 1 (i) $\phi^{k}: A^{k} \times B^{k} \rightarrow \mathbb{R}$ are called convergent continously to $\phi: A \times B \rightarrow \mathbb{R}$ associated with $A^{k} \times B^{k} \stackrel{P-K}{\longrightarrow} A \times B$ if for all $\left(x^{k}, y^{k}\right) \in A^{k} \times B^{k} \rightarrow$ $(x, y), \phi^{k}\left(x^{k}, y^{k}\right) \rightarrow \phi(x, y)$. This convergence is very strong. It implies all the three e/h-, minsup-lop, and maxinf-lop convergences. (Furthermore, the continuous convergence of $\phi$ implies also both the epiconvergnece and hypo-convergence of $\phi^{k}$.) Hence, continuous convergence is a type of variational convergence. But, it is difficult to be satisfied. 
(ii) Similar to the paper ${ }^{16}$ for the finite-dimensionl case, we show in Theorem 5 that in our general case e/h-limits are not unique, but form (epi/hypo) equivalence classes. Fortunately, all the bifunctions in such a class have the same variational properties.

Note that the above definitions of variational convergence of bifunctions do not require that $A^{k} \stackrel{P-K}{\longrightarrow}$ $B^{k} \rightarrow A \times B$. That is why not only points $(x, y) \in A \times B$ are under consideration, but also all the points $(x, y)$, which are limits of $\left(x^{k}, y^{k}\right) \in A^{k} \times B^{k}$, are taken into account.

In order to see the relation between fv-biv $(X \times Y)$ and biv $(X \times Y)$, given $\phi$ in fv-biv $(X \times Y)$, we define the corresponding two so-called proper bifunctions in biv $(X \times Y)$ as follows.

$\left(\eta_{h / e} \phi\right)(x, y):=\left\{\begin{array}{cc}\phi(x, y) & \text { if }(x, y) \in A \times B, \\ +\infty & \text { if } y \in B, x \notin A, \\ -\infty & \text { if } y \notin B .\end{array}\right.$

$\left(\eta_{h / e} \phi\right)(x, y):=\left\{\begin{array}{cr}\phi(x, y) & \text { if }(x, y) \widehat{\mathrm{I}} A \times B, \\ -\infty & \text { if } x \in A, y \notin B, \\ +\infty & \text { if } x \notin A .\end{array}\right.$

Then, we have in fact two "projections" $\eta_{e / h}$ and $\eta_{e / h}$ from fv-biv $(X \times Y)$ to biv $(X \times Y)$ transforming $\phi$ into the two corresponding proper bifunctions belonging to biv $(X \times Y)$. Looking at the definition of e/h-convergence, we see that beside $(x, y) \in A \times$ $B$, we consider also points $(x, y)$ with either $x \in A$ and $y \notin B$ or $x \notin A$ and $y \in B$. We call the points $(x, y) \in A \times B$ and these points the related points (through e/h-convergence). For all the related points, we have $\eta_{e / h}(x, y)=\eta_{h / e}(x, y)$. Because only the related points come into play, we will use the abbreviation $\eta$ for both $\eta_{e / h}$ and $\eta_{h / e}$.

We also need to modify the definition of $\mathrm{e} / \mathrm{h}$ convergence of bifunctions of biv $(X \times Y)$ to have the corresponding Definition 6 below. For $\phi \in$ biv $(X \times Y)$, recall that its domain is defined by $\operatorname{dom} \phi=$ $\operatorname{dom}_{\mathrm{x}} \phi^{\prime} \operatorname{dom}_{\mathrm{y}} \phi$,

where $\operatorname{dom}_{x} \phi:=\{x \in X \mid \phi(x, y)<+\infty, \forall y \in Y\}$, $\operatorname{dom}_{y} \Phi:=\{y \in Y \mid \Phi(x, y)<-\infty, \forall x \in X\}$ Hence, $\phi$ is finite on $\operatorname{dom} \phi$, but $\phi$ also take finite values at different some points outside dom $\phi$.

Definition 6 (epi/hypo-convergence, biv) Let $\phi$ and $\phi^{k}$ be in biv $(X \times Y)$. We say that $\phi^{k} \mathrm{e} / \mathrm{h}$-converge to $\phi$ if

(a') $\forall y \in \operatorname{dom}_{y} \phi, \forall x^{k} \rightarrow x \in X, \exists y^{k} \rightarrow$ $y, l i_{k} \phi^{k}\left(x^{k}, y^{k}\right) \geq \phi(x, y)$;

(b) $\forall x \in \operatorname{dom}_{x} \phi, \forall y^{k} \rightarrow y \in Y, \exists x^{k} \rightarrow$ $x, l s_{k} \phi^{k}\left(x^{k}, y^{k}\right) \geq \phi(x, y)$.
Remark 2 The definition of e/h-convergence ${ }^{8}$ requires that (a') and (b') are fulfilled for all $y \in Y$ and $x \in X$ instead of $y \in d o m_{y} \phi$ and $x \in d o m_{x} \phi$. The following example shows that $\left\{\phi^{k}\right\}_{k}$ e/h-converge in the sense of Definition 6 , but not e/h-converge following ${ }^{8}$. Assume that $X=Y=\mathbb{R}$ and $\phi^{k}$ are defined by

$$
\begin{aligned}
& \phi^{k}(x, y)=\phi(x, y)= \\
& \left\{\begin{array}{c}
0 \text { if }(x, y) \in[1,1]^{2} \\
-\infty \text { if } x \in(0,1), y \in[0,1] \\
+\infty \text { in the other cases. }
\end{array}\right.
\end{aligned}
$$

Then, $\operatorname{dom}_{\mathrm{x}} \phi=(0,1), \operatorname{dom}_{y} \phi=(0,1)$ and $\phi^{k} \mathrm{e} / \mathrm{h}-$ converge to $\phi$ following our definition. However, $\phi^{k}$ do not satisfy (a ) with $\forall y \in \operatorname{dom}_{\mathrm{y}} \phi$ replaced by $\forall y \in$ $Y$. Indeed, for $y=1$ and $x^{k}=k^{-1} \rightarrow 0$, There do not exist $y^{k} \rightarrow-1$ with $l i_{k} \phi^{k}\left(k^{-1}, y^{k}\right) \geq \phi(0,-1)=+\infty$.

\section{CHARACTERIZATIONS OF VARIATIONAL CONVERGENCE OF BIFUNCTIONS}

\section{Characterizations by epi- and hypo- convergence of related unifunctions}

Proposition 1 (characterizations of e/h-convergence by slices)

(i) Condition (a) of e/h-convergence $\Leftrightarrow \forall x^{k} \in A^{k} \rightarrow$ $x$, hli $\phi^{k}\left(x^{k}, \cdot\right) \geq \phi(x, \cdot)$ with $\phi^{k}\left(x^{k}, \cdot\right)$ defined on $A^{k}$ and $\phi(x, \cdot)$ on $A$, and $\forall x^{k} \in A^{k} \rightarrow x \notin A, \exists y^{k} \in$ $B^{k}, \phi^{k}\left(x^{k}, y^{k}\right) \rightarrow+\infty \Leftrightarrow \forall x^{k} \in A^{k} \rightarrow x$, one has (b) of $\phi^{k}\left(x^{k}, \cdot\right) \stackrel{h}{\rightarrow} \phi(x, \cdot)$ with the domains defined as above, and $\forall x^{k} \in A^{k} \rightarrow x \notin A, \exists y^{k} \in B^{k}, \phi^{k}\left(x^{k}, y^{k}\right) \rightarrow+\infty$.

(ii) Condition (b) of e/h-convergence $\Leftrightarrow \forall y^{k} \in B^{k} \rightarrow$ $y$, els $\phi^{k}\left(\cdot, y^{k}\right) \geq \phi(\cdot, y)$ with $\phi^{k}\left(\cdot, y^{k}\right)$ defined on $A^{k}$ and $\phi(\cdot, y)$ on $A$, and $\forall y^{k} \in B^{k} \rightarrow y \notin B, \exists x^{k} \in$ $A^{k}, \phi^{k}\left(x^{k}, y^{k}\right) \rightarrow-\infty \Leftrightarrow \forall y^{k} \in B^{k} \rightarrow y$, one has (a) of $\phi^{k}\left(\cdot, y^{k}\right) \stackrel{e}{\rightarrow} \phi(\cdot, y)$ with the domains defined as above, and $\forall y^{k} \in B^{k} \rightarrow y \notin B, \exists x^{k} \in A^{k}, \phi^{k}\left(x^{k}, y^{k}\right) \rightarrow-\infty$.

Proof (i) We show only the first equivalence. Condition (a) of e/h-convergence is that for all $y \in B$ and $x^{k} \in A^{k} \rightarrow x$, there exist $y^{k} \in B^{k} \rightarrow y$ such that $l i_{k} \phi^{k}\left(x^{k}, y^{k}\right) \geq \phi(x, y)$ when $x \in A$ together with the upper hypo-limit defined in part II. Moreover, the infinity condition in (a) of e/h-convergence is just the rest in the second side of this first equivalence. Similarly, applying this condition (a) together with the definition of hypo-convergence, we obtain the second equivalence.

(ii) The arguments are similar to those in (i) due to the symmetric property of epi/hypo-convergence.

Proposition 2 (characterizations of minsup-lop convergence by slices) 
(i) Condition (a) of misup-lop convergence is the same (a) of epi/hypo-convergence and so we have the same two equivalences in Proposition 1

(ii) Condition (b) of minsup-lop convergence $\Leftrightarrow \forall x \in A, \exists x^{k} \in A^{k} \rightarrow x$, such that $h l s \phi^{k}\left(x^{k}, \cdot\right) \leq \phi\left(x^{k}, \cdot\right)$ with $\phi\left(x^{k}, \cdot\right)$ defined on $B$ and $\min _{\left\{y^{k} \rightarrow y\right\}} \lim _{k} \phi^{k}\left(x^{k}, y^{k}\right)=-\infty$ when $y \in B$ Proof We need to prove only (ii). Condition (b) of minsup-lop convergence is $\forall x \in A, \exists x^{k} \in A^{k} \rightarrow x$, $\forall y^{k} \in B^{k} \rightarrow y, l s_{k} \phi^{k}\left(x^{k}, y^{k}\right) \leq \phi(x, y)$ if $y \in B$ and $\phi^{k}\left(x^{k}, y^{k}\right) \rightarrow-\infty$ if $y \notin B$ Combining this with the definition of lower hypo-limits, we complete the proof.

Corollary 1: Let $\phi^{k} \stackrel{\text { mins }}{\longrightarrow} \phi$.

(i) There exist $x^{k} \in A^{k} \rightarrow x \in A$ such that $\phi\left(x^{k}, \cdot\right) \stackrel{h}{\rightarrow}$ $\phi(x, \cdot)$ on $B$ and $\max _{\left\{y^{k} \in B^{k} \rightarrow y \in B\right\}} \lim _{k} \phi\left(x^{k}, y^{k}\right)=$ $+\infty$ for all $x^{k} \in A^{k} \rightarrow x \notin A$;

(ii) If $\phi^{k}$ do not depend on y (x, resp), then $\phi\left(x^{k}, \cdot\right) \stackrel{e}{\rightarrow}$ $\phi(x, \cdot)\left(\phi\left(\cdot, y^{k}\right) \stackrel{h}{\rightarrow} \phi(\cdot, y)\right.$, resp $)$.

Remark 3 (i) The characterization of maxinf-lop convergence in terms of slices clearly states as follows: for $\phi^{k} \stackrel{\text { maxi }}{\longrightarrow} \phi$,

(a) there exist $y^{k} \in B^{k} \rightarrow y \in B$ such that $\phi\left(\cdot, y^{k}\right) \stackrel{e}{\rightarrow} \phi(\cdot, y)$ on $A$ and $\min$ $\min _{\left\{\mathrm{x}^{k} \in A^{k} \rightarrow x \in A\right\}} \lim _{k} \phi\left(x^{k}, y^{k}\right)=-\infty$ for all $y^{k} \in B^{k} \rightarrow y \notin B$

(b) when $\phi^{k}$ do not depend on $x$ (on $y$ ), $\phi(\cdot, \cdot) \stackrel{h}{\rightarrow}$ $\phi(\cdot, \cdot)\left(\phi\left(x^{k}, \cdot\right) \stackrel{e}{\rightarrow} \phi(x, \cdot)\right.$, resp $)$., resp).

(ii) We also have an assertion corresponding to (i) in Corollary 1 , but with attention on changes on the roles of $(x, y)$ and of epi- and hypo-convergence. However, there is no direct connection between the lopconvergence of $\phi^{k}$ to $\phi$ and $\phi(\cdot, y) \stackrel{e}{\rightarrow} \phi(\cdot, y)$ for all $y$ and $\phi(x, \cdot) \stackrel{h}{\rightarrow} \phi(x, \cdot)$ for all $x$. If $\phi^{k}$ depend only on one component, (ii) in Corollary 1 becomes a sufficient condition for the lop-convergence to $\phi$.

Next, we discuss a relation between lop-convergence of bifunctions and convergence of other unifunctions being its sup- and inf-projections defined as follows. The sup-projection (inf-projection, resp) of $\phi \in \mathrm{fv}$ biv $(X \times Y)$ is $\zeta: A \rightarrow \mathbb{R} \cup\{+\infty\}(\eta: B \rightarrow \mathbb{R}$, resp) defined by, $\zeta(x):=\sup _{y \in B} \phi(x, y)(\eta(y):=$ $\inf _{\mathrm{x} \in \mathrm{A}} \phi(x, y)$, resp).

To establish this relation, we need the following concept of ancillary tight convergence.

Definition 7 (ancillary tight $\mathrm{e} / \mathrm{h}$ - and lopconvergence)

(i) (x-ancillary tight e/h-convergence) $\phi^{k}$ are called $\mathrm{x}$ ancillary e/h-convergent to $\phi$ if (a) of e/h-convergence and the following condition are satisfied

(be) $\forall y \in B, \forall x \in A, \forall y^{k} \in B^{k} \rightarrow y, \exists x^{k} \in A^{k} \rightarrow x$, $\operatorname{ls}_{k} \zeta^{k}\left(x^{k}\right) \leq \zeta(x)$. (ii) (y-ancillary tight e/h-convergence) $\phi^{k}$ are called $y$-ancillary e/h-convergent to $\phi$ if (b) of e/h-convergence is fulfilled together with the condition

(ae) $\forall x \in A, y \in B, \forall x^{k} \in A^{k}, \exists y^{k} \in B^{k} \rightarrow$ $y, \mathrm{li}_{k} \eta^{k}\left(y^{k}\right) \geq \eta(y)$.

(iii) (-ancillary tight minsup-lop convergence) $\phi^{k}$ are called $\mathrm{x}$-ancillary tightly minsup-lop convergent to $\phi$ if one has (a) of minsup-lop convergence and (b) strenghthened as

(b1) condition (b) holds and, for any positive $\varepsilon$ and any $x^{k} \in A^{k} \rightarrow x$ given in (b), there exist a compact set $B_{e} \subset Y$ and $k_{\varepsilon}$ such that $\sup _{B_{\varepsilon} \subset B^{k}} \phi^{k}\left(x^{k}, \ldots\right) \geq$ $\sup _{B^{k}} \phi^{k}\left(x^{k}, \ldots\right)-\varepsilon, \forall k \geq k_{\varepsilon}$.

(iv) (y-ancillary tight maxninf-lop convergence) $\phi^{k}$ are said to $y$-ancillary tight maxinf-lop converge to $\phi$ if if one has (a) of maxinf-lop convergence and (b) strenghthened as

(b2) Condition (b) holds and, for any positive $\varepsilon$ and any $y^{k} \in B^{k} \rightarrow y$ given in (b), there exists a compact set $A_{\varepsilon} \subset X$ and $k_{\varepsilon}$ such thatinf ${ }_{A_{\varepsilon} \cap A^{k}} \phi^{k}(\ldots, y) \leq$ $\sup _{A^{k}} \phi^{\mathrm{k}}\left(\ldots, y^{k}\right)+\varepsilon, \forall k \geq k_{\varepsilon}$.

Proposition 3 (x-ancillary tight e/h-convergence and epi-convergence of sup-projections) Let the e/hconvergence of $\phi^{k}$ to $\phi$ in fv-biv $(X \times Y)$ be $\mathrm{x}$ ancillary tight and $\zeta^{k}, \zeta$ be sup-projections of $\phi^{k}, \phi$, resp, such that $\operatorname{dom} \zeta \neq 0$ and $\operatorname{dom} \zeta^{k} \neq 0$. Then, $\mathrm{e}-\lim _{k} \zeta^{k}=\zeta$.

Proof To check condition (a) of epi-convergence for $\zeta^{k}$, let $x^{k} \in \operatorname{dom} \zeta^{k} \rightarrow x$. First, assume that $x^{k} \in$ $\operatorname{dom} \zeta^{k}$. For each $\varepsilon>0$, choose $y_{\varepsilon} \in B$ such that $\phi\left(x, y_{\varepsilon}\right) \geq \xi(x)-\varepsilon$. By (a) in Definition 4 , there are $y_{\varepsilon}^{k} \in B^{k} \rightarrow y_{\varepsilon}$ such that

$\operatorname{li}_{k} \zeta^{k}\left(x^{k}\right) \geq \operatorname{li}_{k} \phi\left(x^{k}, y^{k}\right) \geq \phi\left(x, y_{\varepsilon}\right) \geq \zeta(x)-\varepsilon$. As $\varepsilon$ is arbitrary, $\operatorname{li}_{k} \zeta^{k}\left(x^{k}\right) \geq \zeta(x)$..

If $\zeta(x)=+\infty$, for each $\gamma>0$, choose $y_{\gamma} \in B$ such that $\phi\left(x, y_{\gamma}\right) \geq \gamma$. Again by the above (a), there are $y_{\gamma}^{k} \in B^{k} \rightarrow y_{\gamma}$ with $\operatorname{li}_{k} \zeta^{k}\left(x^{k}\right) \geq \operatorname{li}_{k} \phi\left(x^{k}, y_{\gamma}^{k}\right) \geq \gamma$. By the arbitrariness of $\gamma, \zeta^{k}\left(x^{k}\right) \rightarrow+\infty$.

To verify condition (b) of the epi-convergence, for any $x^{k} \widehat{\text { Idom } \zeta}$ take arbitrarily $y^{k} \in B^{k} \rightarrow y \in B$. By condition (be), there exist $x^{k} \in A^{k} \rightarrow x$ such that $\operatorname{ls}_{k} \zeta^{k}\left(x^{k}\right) \leq \zeta(x)$. Hence, for some $k_{0}$ and all $k \geq k_{0}$, $\zeta^{k}\left(x^{k}\right)$ is finite, i.e., $x^{k} \in \operatorname{dom} \zeta^{k}$ and so the aforementioned (b) is fulfilled.

Proposition 4 ( $x$-ancillary tight minsup-lop convergence and epi-convergence of sup-projections) Assume that $\phi^{k}$ minsup-lop converge $\mathrm{x}$-ancillary tightly to $\phi, \operatorname{dom} \zeta \neq 0$ and $\operatorname{dom} \zeta^{k} \neq 0$. Then, $\zeta^{k}$ epiconverge to $\zeta$.

Proof We prove the equivalent conclusion: epi $\zeta^{k} \stackrel{P-K}{\longrightarrow}$ epi $\zeta$. To see $\operatorname{Ls}_{k}\left(\right.$ epi $\left.\zeta^{k}\right) \subset$ epi $\zeta$, take 
any $(x, \alpha)$ in the left-hand side, which means there are $k_{j}$ such that $\left(x^{k_{j}}, \alpha^{k_{j}}\right) \in \mathrm{epi} \zeta^{\mathrm{k}_{\mathrm{j}}}$ tend to $(x, \alpha)$. We claim that $x \in \operatorname{dom} \zeta$. Clearly, $x \in A$, because otherwise condition (a) of minsup-lop convergence yields $y^{k_{j}} \in B^{k_{j}}$ such that $\phi^{k_{j}}\left(x^{k_{j}}, y^{k_{j}}\right) \rightarrow+\infty$, which contradicts the fact that $\alpha^{k_{j}} \geq \sup _{B^{k_{j}}} \phi^{k_{j}}\left(x^{k_{j}}, \cdot\right)$ and $\alpha^{k_{j}} \rightarrow \alpha$. Suppose $\zeta(x)=+\infty$. Then, for any $\gamma>\alpha$, there exists $y_{\gamma} \in B$ with $\phi\left(x, y_{\gamma}\right)>\gamma$. In view of the above (a), there exist $y_{\gamma}^{k_{j}} \in B^{k_{j}} \rightarrow y_{\gamma}$ such that $\operatorname{li}_{j} \phi^{k_{j}}\left(x^{k_{j}}, y^{k_{j}}\right) \geq \phi\left(x, y_{\gamma}\right)$. Hence, $\alpha=\lim _{j} \alpha^{k_{j}} \geq \operatorname{li}_{j} \zeta^{k_{j}}\left(x^{k_{j}}\right) \geq \operatorname{li}_{j} \phi^{k_{j}}\left(x^{k_{j}}, y^{k_{j}}\right) \geq \phi\left(x, y_{\gamma}\right)>$ $\gamma>\alpha$. This contradiction shows that $x \in \operatorname{dom} \zeta$.

For any $\varepsilon>0$, there exists $y_{\varepsilon} \in B$ with $\phi\left(x, y_{\varepsilon}\right) \geq$ $\zeta(x)-\varepsilon$. The aforementioned (a) again gives $y_{\varepsilon}^{k_{j}} \in$ $B^{k_{j}} \rightarrow y_{\varepsilon}$ such that

$\operatorname{li}_{j} \zeta^{k_{j}}\left(x^{k_{j}}\right) \geq \operatorname{li}_{j} \phi^{k_{j}}\left(x^{k_{j}}, y^{k_{j}}\right) \geq \phi\left(x, y_{\varepsilon}\right) \geq \zeta(x)-\varepsilon$.

Therefore, as $\varepsilon$ is arbitrary,

$\alpha=\lim _{j} \alpha^{k_{j}}=\operatorname{li} \zeta^{k_{j}}\left(x^{k_{j}}\right) \geq \zeta(x)$.

So, $\operatorname{Ls}_{k}\left(\right.$ epi $\left.\zeta^{k}\right) \subset$ epi $\zeta$. To prove the inclusion epi $\zeta \subset$ $\mathrm{Li}_{k}$ epi $\zeta^{k}$, observe that $\phi^{k}$ minsup-lop convergent $\mathrm{x}$ ancillary tightly to $\phi$ implies that for any $x \in A$, there exists $x^{k} \in A^{k}$ ensuring the tight hypo-convergence of $\phi^{k}\left(x^{k}, \cdot\right)$ to $\phi(x, \cdot)$. Using the counterpart of Theorem 2 (i) for hypo-convergence, one has $\zeta^{k}\left(x^{k}\right) \rightarrow \zeta(x)$. For any $(x, \alpha) \in$ epi $\zeta$, i.e., $\alpha \geq \zeta(x)$, clearly, $\left(x^{k}, \zeta^{k}\left(x^{k}\right)+\alpha-\zeta(x)\right) \in$ epi $\zeta^{k} \rightarrow(x, \alpha)$. Therefore, epi $\zeta \subset \operatorname{Li}_{k}$ epi $\zeta^{k}$.

We omit the statements about inf-projections corresponding to the above propositions.

\section{Characterizations by proper bifunctions}

Theorem 3 For $\phi, \phi^{k}$ in fv-biv $(X \times Y), \phi^{k} \mathrm{e} / \mathrm{h}$ converge to $\phi$ if and only if $\eta \phi^{k} \mathrm{e} / \mathrm{h}$-converge to $\eta \phi$. Proof We use the explicit formula of $\eta_{e / h}$, but denotes it by $\eta$ for simplicity.

(a') of Definition $6 \Rightarrow$ (a) of Definition 4. Suppose $y \in$ $B=\operatorname{dom}_{y}(\eta \phi)$ and $x^{k} \in A^{k} \rightarrow x$. Then, (a') yields $x^{k} \in$ $A^{k} \rightarrow x$ such that

$$
\operatorname{li}_{k}\left(\eta \phi^{k}\right)\left(x^{k}, y^{k}\right) \geq(\eta \phi)(x, y) .
$$

Consider first $x \in A$. Suppose the existence of $y^{k_{j}} \notin B^{k_{j}}$. Then, $\left(\eta \phi^{k_{j}}\right)\left(x^{k_{j}}, y^{k_{j}}\right)=-\infty$, contradicting (1). Hence, $y^{k} \in B^{k}$ and (1) means that $\operatorname{li}_{k} \phi^{k}\left(x^{k}, y^{k}\right) \geq \phi(x, y)$ as (a) requires. Now let $x \notin$ $A$. Then, $(\eta \phi)(x, y)=+\infty$ and (1) implies that $\left(\eta \phi^{k}\right)\left(x^{k}, y^{k}\right) \rightarrow+\infty$. Again $y^{k_{j}} \notin B^{k_{j}}$ would give $\left(\eta \phi^{k_{j}}\right)\left(x^{k_{j}}, y^{k_{j}}\right) \equiv-\infty$, contradicting (1). Hence, $\phi^{k}\left(x^{k}, y^{k}\right) \equiv\left(\eta \phi^{k}\right)\left(x^{k}, y^{k}\right) \rightarrow+\infty$ as (a) requires.

(a) $\Rightarrow$ (a). Let $y \in B=\operatorname{dom}_{\mathrm{y}}(\eta \phi)$ and $x^{k} \rightarrow x \in$ $X$. If $x \in A$ and there exist $x^{k_{j}} \in A^{k_{j}}$, then (a) give $y^{k_{j}} \in B^{k_{j}} \rightarrow y$ such that $l i_{j}\left(\eta \phi^{k_{j}}\right)\left(x^{k_{j}}, y^{k_{j}}\right)=$ $l i i_{j}\left(\eta \phi^{k_{j}}\right)\left(x^{k_{j}}, y^{k_{j}}\right) \geq(\eta \phi)(x, y)$.
We build a sequence $y^{k}$ by inserting, for $k$ with $x^{k} \notin$ $A^{k}, y^{k} \in B^{k}$ so that $y^{k} \rightarrow y$. Then, for $k$ with $x^{k} \notin A^{k}$, $\left(\eta \phi^{k}\right)\left(x^{k}, y^{k}\right) \rightarrow+\infty$ and does not effect the liminf in (2). Hence, we have (1). In the case that $x^{k} \notin A^{k}$, we can take any $y^{k} \in B^{k} \rightarrow y$ to obtain $\left(\eta \phi^{k}\right)\left(x^{k}, y^{k}\right) \rightarrow$ $+\infty$ and then get (1).

If $x^{k} \notin A^{k}$, as $y \in B$ one has $(\eta \phi)(x, y) \rightarrow+\infty$. If, for the given $\left\{x^{k}\right\}_{k}, x^{k} \notin A^{k}$, we take arbitrarily $y^{k} \in$ $B^{k} \rightarrow y$ to have $\left(\eta \phi^{k}\right)\left(x^{k}, y^{k}\right) \rightarrow+\infty$ and get (1). Otherwise, there is a subsequence $x^{k_{j}} \in A^{k_{j}}$. Then, (a) yields $y^{k_{j}} \in B^{k_{j}} \rightarrow y$ such that $\phi^{k_{j}}\left(x^{k_{j}}, y^{k_{j}}\right) \rightarrow+\infty$. For the additional $k$ with $x^{k} \notin A^{k}$, we add to $\left\{y^{k_{j}}\right\}$ points $y^{k} \in B^{k}$ so that $y^{k} \rightarrow y$. Because, for additional $k,\left(\eta \phi^{k}\right)\left(x^{k}, y^{k}\right) \rightarrow+\infty$, we have the entire convergent sequence $\phi^{k}\left(x^{k}, y^{k}\right) \rightarrow+\infty$ and so (1).

(b) $\Rightarrow$ (b') Let $x \in A$ and $y^{k} \in B^{k} \rightarrow y$. (b') supplies $x^{k} \rightarrow x$ such that

$$
\operatorname{ls}_{k}\left(\eta \phi^{k}\right)\left(x^{k}, y^{k}\right) \leq(\eta \phi)(x, y)
$$

If $y \in B_{k}$ and there is a subsequence $x^{k_{j}} \notin A^{k_{j}}$, then $\phi^{k_{j}}\left(x^{k_{j}}, y^{k_{j}}\right) \equiv+\infty$ contradicting (3). Thus, $x^{k} \in A^{k}$ as (b) requires. If $x^{k} \in A^{k}$, then (3) implies that $\operatorname{ls}_{k}\left(\eta \phi^{k}\right)\left(x^{k}, y^{k}\right) \leq \phi(x, y)$ as (b) requires. If $y \notin B$, $(\eta \phi)(x, y)=+\infty$. Hence, we get a contradiction if there is a subsequence $x^{k_{j}} \notin A^{j}$. Therefore, $x^{k} \in A^{k}$ and we obtain (b).

(b) $\Rightarrow$ (b'). This is similar to (a) $\Rightarrow$ (a') combined with $\left(b^{\prime}\right) \Rightarrow(b)$.

\section{Geometric characterizations}

To characterize e/h- and lop-convergence of $\left\{\phi^{k}\right\}_{k}$ to $\phi$ in a geometric way, we introduce the following six limit bifunctions of $\left\{\phi^{k}\right\}_{k}$ in connection with $\phi$.

Definition 8 (six limit bifunctions) Let $\phi$ and $\phi^{k}$ be in fv-biv $(X \times Y)$ and $(x, y) \in X \times Y$.

(i) (lower lop-limit bifunctions)

$\operatorname{lli} \phi^{k}(x, y):=\sup _{\left\{y^{k} \in B^{k} \rightarrow y\right\}} \inf _{\left\{x^{k} \in A^{k} \rightarrow x\right\}} \operatorname{li}_{k} \phi^{k}\left(x^{k}, y^{k}\right)$ for $y \in B$,

$\operatorname{lli} \phi^{k}(x, y):=\sup _{\left\{y^{k} \in B^{k} \rightarrow y\right\}} \inf _{\left\{x^{k} \in A^{k} \rightarrow x\right\}} \operatorname{ls}_{k} \phi^{k}\left(x^{k}, y^{k}\right)$ if $y \notin B$

(ii) (upper lop-limit bifunctions)

$\operatorname{lls} \phi^{k}(x, y):=\inf _{\left\{x^{k} \in A^{k} \rightarrow x\right\}} \sup _{\left\{y^{k} \in B^{k} \rightarrow y\right\}} \operatorname{ls}_{k} \phi^{k}\left(x^{k}, y^{k}\right)$ for $x \in A$,

$\operatorname{lls} \phi^{k}(x, y):=\inf _{\left\{x^{k} \in A^{k} \rightarrow x\right\}} \sup _{\left\{y^{k} \in B^{k} \rightarrow y\right\}} \operatorname{li}_{k} \phi^{k}\left(x^{k}, y^{k}\right)$ if $x \notin A$.

(iii) (lower and upper e/h-limit bifunctions)

$\operatorname{ehli} \phi^{k}(x, y):=\sup _{\left\{y^{k} \in B^{k} \rightarrow y\right\}} \inf _{\left\{x^{k} \in A^{k} \rightarrow x\right\}} l_{k} \phi^{k}\left(x^{k}, y^{k}\right)$ if $y \in B, x \in A$,

$\operatorname{ehls} \phi^{k}(x, y):=\inf _{\left\{x^{k} \in A^{k} \rightarrow x\right\}} \sup _{\left\{y^{k} \in B^{k} \rightarrow y\right\}} \operatorname{li}_{k} \phi^{k}\left(x^{k}, y^{k}\right)$ if $x \in A, y \in B$.

Remark 4 Straightforwardly from the definitions one always has 
$\operatorname{ehls} \phi^{k}(x, y) \leq \operatorname{lls} \phi^{k}(x, y)$ for $x \in A, y \in B$

$\operatorname{lli} \phi^{k}(x, y) \leq \operatorname{ehli} \phi^{k}(x, y)$ for $x \in A, y \in B$

In Theorem 6 below, we will see that, just due to these inequalities, both minsup-lop and maxinf-lop limits are unique if exist. Moreover, one also has $\operatorname{lli} \phi^{k}(x, y) \leq \operatorname{lls} \phi^{k}(x, y)$ for $(x, y) \in A \times B$ $\operatorname{ehls} \phi^{k}(x, y) \leq \operatorname{lls} \phi^{k}(x, y)$ for $(x, y) \in A \times B$ $\operatorname{lli} \phi^{k}(x, y) \leq \operatorname{ehls} \phi^{k}(x, y)$ for $(x, y) \in A \times B$

Theorem 4 (formulas of the six limit bifunctions) Let $\phi$ and $\phi^{k}$ be in fv-biv $(X x Y)$ and $(x, y) \in X \times Y$.

(i) (lower lop-limit bifunctions)

$\operatorname{lii} \phi^{k}(x, y):=\max _{\left\{y^{k} \in B^{k} \rightarrow y\right\}} \inf _{\left\{x^{k} \in A^{k} \rightarrow x\right\}} \operatorname{li}_{k} \phi^{k}\left(x^{k}, y^{k}\right)$ for $y \in B$,

$\operatorname{lli} \phi^{k}(x, y):=\sup _{\left\{y^{k} \in B^{k} \rightarrow y\right\}} \min _{\left\{x^{k} \in A^{k} \rightarrow x\right\}} \operatorname{ls}_{k} \phi^{k}\left(x^{k}, y^{k}\right)$ if $y \notin B$,

(ii) (upper lop-limit bifunctions)

$\operatorname{lls} \phi^{k}(x, y):=\min _{\left\{x^{k} \in A^{k} \rightarrow x\right\}} \sup _{\left\{y^{k} \in B^{k} \rightarrow y\right\}} 1 \mathrm{~s}_{k} \phi^{k}\left(x^{k}, y^{k}\right)$ for $x \in A$,

$\operatorname{lls} \phi^{k}(x, y):=\inf _{\left\{x^{k} \in A^{k} \rightarrow x\right\}} \max _{\left\{y^{k} \in B^{k} \rightarrow y\right\}} \operatorname{li}_{k} \phi^{k}\left(x^{k}, y^{k}\right)$ if $x \notin A$.

(iii) (lower and upper e/h-limit bifunctions)

ehli $\phi^{k}(x, y):=\sup _{\left\{y^{k} \in B^{k} \rightarrow y\right\}} \min _{\left\{x^{k} \in A^{k} \rightarrow x\right\}} \operatorname{ls}_{k} \phi^{k}\left(x^{k}, y^{k}\right)$ if $y \in B, x \in A$,

$\operatorname{ehls} \phi^{k}(x, y)=\inf _{\left\{x^{k} \in A^{k} \rightarrow x\right\}} \max _{\left\{y^{k} \in B^{k} \rightarrow y\right\}} \operatorname{li}_{k} \phi^{k}\left(x^{k}, y^{k}\right)$ if $x \in A, y \in B$.

Proof For reasons of similarity, we discuss only (ii). To prove the first formula, denote $u:=1 l s \phi^{k}(x, y)$. If $u=+\infty$, any $\left\{x^{k} \in A^{k} \rightarrow x\right\}$ is a minimizer for the considered expression. If $-\infty<u<+\infty$, for any $\varepsilon^{j} \searrow 0$, there exist $x_{j}^{k} \in A^{k} \rightarrow x$ such that for all $y_{j}^{k} \in B^{k} \rightarrow y, \operatorname{ls}_{k} \phi^{k}\left(x_{j}^{k}, y_{j}^{k}\right) \leq u+\varepsilon^{j}$.. We take $\mathrm{ls}_{j}$ to get $\operatorname{ls}_{j} 1 \mathrm{~s}_{k} \phi^{k}\left(x_{j}^{k}, y_{j}^{k}\right) \leq u$. Due to Corollary A3 in [7], for each $k$ there is $j(k)$ such that $1 s_{k} \phi^{k}\left(x_{j(k)}^{k}, y_{j(k)}^{k}\right) \leq u$. Hence, the sequence $\left\{x_{j(k)}^{k}\right\}_{k}$ is a minimizer. If $u=$ $-\infty$, for any $\theta^{j} \rightarrow+\infty$, one finds $x_{j}^{k} \in A^{k} \rightarrow x$ such that for all $y_{j}^{k} \in B^{k} \rightarrow y, \operatorname{ls}_{k} \phi^{k}\left(x_{j}^{k}, y_{j}^{k}\right) \leq-\theta^{j}$. Similarly as above, with that corollary we see that the obtained $\left\{x_{j(k)}^{k}\right\}_{k}$ is a minimizer.

Consider the second formula. Fix a sequence $\left\{x^{k} \in A^{k}\right\}_{k} \rightarrow A$ and denote

$s:=\sup _{\left\{y^{k} \in B^{k_{j}}\right\}} \operatorname{li}_{k} \phi^{k}\left(x^{k}, y^{k}\right)$

We check the achievement of the involved supremum. If $s=-\infty$, any $\left\{y^{k} \in B^{k}\right\}_{k}$ gives the maximum. If $-\infty<s<+\infty$, for any $\varepsilon^{j} \searrow 0$, there exist $y^{k} \in B^{k}$ with $\operatorname{li}_{k} \phi^{k}\left(x^{k}, y_{j}^{k}\right) \geq s-\varepsilon^{j}$ for each $j \in$ $\mathbb{N}$. Taking $\operatorname{li}_{j}$ we have $\operatorname{li}_{j} \mathrm{li}_{k} \phi^{k}\left(x^{k}, y_{j}^{k}\right) \geq s$. In view of Lemma A. ${ }^{8}$, we have $j(k)$ for each $k$ such that $\operatorname{li}_{k} \phi^{k}\left(x^{k}, y_{j(k)}^{k}\right) \geq s$. Of course the sequence $\left\{y_{j(k)}^{k}\right\}_{k}$ gives the aforementioned maximum. By the arbitrariness of $\left\{x^{k}\right\}_{k}$, we obtain the required equality. If $s=+\infty$, then for any $\theta^{j} \rightarrow+\infty$, one finds $y_{j}^{k} \in B^{k}$ such that $\operatorname{li}_{k} \phi^{k}\left(x^{k}, y_{j}^{k}\right) \geq \theta^{j}$ for any $j$. Taking $\mathrm{li}_{j}$, one arrives at $\operatorname{li}_{j} l_{k} \phi^{k}\left(x^{k}, y_{j}^{k}\right)=+\infty$. Again by Lemma A. $1^{8}$, one gets $j(k)$ for each $k$ such that $\operatorname{li}_{k} \phi^{k}\left(x^{k}, y_{j(k)}^{k}\right)=$ $+\infty$. Hence, $\left\{y_{j(k)}^{k}\right\}_{k}$ is a maximizer for the supexpression in the definition of $s$.

Theorem 5 (characterization of e/h-convergence) Let $\phi$ and $\phi^{k}$ be in fv-biv $(X \times Y)$. Then, $\phi^{k}$ e/h-converge to $\phi$ if and only if the following two assertions hold

(a) $\phi(x, y) \leq \operatorname{ehls} \phi^{k}(x, y)$ when $x \in A$ and $y \in B$, and lls $\phi^{k}(x)=+\infty$ when $x \notin A$;

(b) ehli $\phi^{k}(x, y) \leq \phi(x, y)$ when $y \in B$ and $x \in A$, and $\operatorname{lli} \phi^{k}(x)=+\infty$ when $y \notin B$.

Proof From Definition 4 and this theorem, it is obvious that (a) and (b) of e/h-convergence coincide with (a) and (b), resp, of Theorem 5 .

Observe that for $\phi^{k}$ fv-biv $(X \times Y)$, which of ehli $\phi^{k}(x, y)$ and ehls $\phi^{k}(x, y)$ is smaller changes from point to point in general. Theorem 5 stipulates that if $\operatorname{ehli} \phi^{k}(x, y) \leq \operatorname{ehls} \phi^{k}(x, y)$ for all $(x, y) \in A \times B$, any $\phi$ satisfying the inequality ehli $\phi^{k}(x, y) \leq \phi \leq$ ehls $\phi^{k}(x, y)$ on $A \times B$ and fulfills also the infinity items (for $x$ outside $A$ or y outside $B$ ) is an e/h-limit. Thus, in general the limits of a given e/h-convergent sequence, if exist, form an e/h-equivalence class of bifunctions.

Theorem 6 (characterizations of lop-convergence) Let $\phi$ and $\phi^{k}$ be in fv-biv $(X \times Y)$.

(i) $\phi^{k}$ minsup-lop converge to $\phi$ if and only if: for $x \in A, \operatorname{lls} \phi^{k}(x, y) \leq \phi(x, y) \leq \operatorname{ehls} \phi^{k}(x, y)$ if $y \in B$, and $\operatorname{lls} \phi^{k}(x, y)=-\infty$ if $y \notin B$, and for $x \notin A$, $1 \mathrm{ls} \phi^{k}(x)=$ $+\infty$. Hence, we have in fact the equalities for $(x, y) \in$ $A \times B$ by Remark 4 .

(ii) $\phi^{k}$ maxinf-lop converge to $\phi$ if and only if: for $y \in B$, ehli $\phi^{k}(x, y) \leq \phi(x, y) \leq \operatorname{lli} \phi^{k}(x, y)$ if $x \in A$, and $\operatorname{lli} \phi^{k}(x, y)=+\infty$ if $x \notin A$, and for $y \notin B$, 11 il $\phi^{k}(y)=$ $-\infty$. Hence, we have in fact the equalities for $(y, x) \in$ $A \times B$ by Remark 4 .

Proof (i) Following the second formula in Theorem 4 (ii) and (iii), condition (a) of minsup-lop convergence means that $\phi(x, y) \leq \operatorname{ehls} \phi^{k}(x, y)$ if $x \in A, y \in B$, and $\operatorname{lli} \phi^{k}(x)=+\infty$ if $x \notin A$, which is a part of assertion (i). For $x \in A$ and $y \widehat{\mathrm{I}} Y$, take any $\varepsilon^{j} \searrow 0$ and $\gamma^{j} \nearrow+\infty$. We define

$$
\theta^{j}(x, y):= \begin{cases}\phi(x, y)+\varepsilon^{j} \text { if } y \in B, \\ -\gamma^{j} & \text { if } y \notin B .\end{cases}
$$

The remaining part of (i) means that, for $x \in A$ and $y \in Y$,

$$
\operatorname{lls} \phi^{k}(x, y) \leq\left\{\begin{array}{l}
\phi(x, y) \text { if } y \in B \\
-\infty \text { if } y \notin B .
\end{array}\right.
$$


This is equivalent to the statement: for each $j$, there exist $x_{j}^{k} \in A^{k} \rightarrow x$, for all $y_{j}^{k} \in B^{k} \rightarrow y, \operatorname{ls}_{k} \phi^{k}\left(x_{j}^{k}, y_{j}^{k}\right) \leq$ $\theta^{j}(x, y)$. Taking $\mathrm{ls}_{j}$ of both sides yields

$$
\operatorname{ls}_{j} \operatorname{ls}_{k} \phi^{k}\left(x_{j}^{k}, y_{j}^{k}\right) \leq\left\{\begin{array}{l}
\phi(x, y) \text { if } y \in B, \\
-\infty \text { if } y \notin B .
\end{array}\right.
$$

By virtue of Corollary A3 in ${ }^{8}$, one has $j(k)$ for each $k$ such that

$$
\operatorname{ls}_{k} \phi^{k}\left(x_{j(k)}^{k}, y_{j(k)}^{k}\right) \leq\left\{\begin{array}{l}
\phi(x, y) \text { if } y \in B, \\
-\infty \text { if } y \notin B .
\end{array}\right.
$$

Therefore, the sequence $\left\{x^{k}\right\}_{k}:=\left\{x_{j(k)}^{k}\right\}_{k}$ is a minimizer for the expression of lls $\phi^{k}(x, y)$, i.e., we obtain the remaining part of assertion (i).

(ii) The proof is similar to (i), but applying Theorem 4 with the first formula of (iii), both the formulas of (i), and Lemma $\mathrm{A} 1^{8}$ instead of the formulas of Theorem 4 applied in (i) and Corollary A37, resp.

By Theorem 6, we see that, if exists, each of the minsup-lop limit and maxinf-lop limit is unique. Furthermore, both minsup-lop and maxinf-lop limits exist at the same time if and only if, for $(x, y) \in A \times B$, $\operatorname{lli} \phi^{k}=\operatorname{ehli} \phi^{k}=\phi=\operatorname{ehls} \phi^{k}=11 s \phi^{k}$

together with the four infinity conditions stated in Theorem 6.

\section{ACKNOWLEDGMENTS}

This research was supported by the Project of Vietnam national University of type $\mathrm{C}$ under grant number C2019-20-05. I am deeply indebted to the anonymous reviewer for the valuable remarks and suggestions, which helped me so much in completing this final version.

\section{CONFLICT OF INTERESTS}

The author declares that there is no conflict of interest in publishing this paper.

\section{AUTHORS' CONTRIBUTIONS}

This study is finished by one author.

\section{REFERENCES}

1. Walkup DW, Wets RJB. Continuity of some convex-conevalued mappings. Proc Amer Math Soc. 1967;18:229235. Available from: https://doi.org/10.1090/S0002-99391967-0209806-6.

2. Wijsman RA. Convergence of sequences of convex sets, cones and functions. Bull Amer Math Soc. 1964;70:186-188. Available from: https://doi.org/10.1090/S0002-9904-1964-11072-7.

3. Wijsman RA. Convergence of sequences of convex sets, cones and functions II. Trans Amer Math Soc. 1966;123:32-45. Available from: https://doi.org/10.1090/S0002-9947-1966-01965998.

4. Attouch $\mathrm{H}$. Variational Convergence for Functions and Operators, Applicable Mathematics Series. Pitman. 1984;.

5. Aubin JP, Frankowska H. Set-Valued Analysis, Birkhäuser, Boston. 1990;
6. Rockafellar RT, Wets RJB. Variational Analysis. Springer, 3rd printing edition. 2009;.

7. Attouch $\mathrm{H}$, Wets RJB. Approximation and convergence in nonlinear optimization, in Nonlinear Programming, O. Mangasarian, R. Meyer and S. Robinson, eds. Academic Press, New York. 1981;p. 367-394. Available from: https://doi.org/ 10.1016/B978-0-12-468662-5.50018-5.

8. Attouch $\mathrm{H}$, Wets RJB. A convergence theory for saddle functions. Trans Amer Math Soc. 1983;280:1-41. Available from: https://doi.org/10.1090/S0002-9947-1983-0712247-X.

9. Attouch $\mathrm{H}$, Wets RJB. A convergence for bivariate functions aimed at the convergence of saddle values. Mathematical Theory Optimization, J Cecconi and T Zolezzi, eds, Springer. 1983;p. 1-42. Available from: https://doi.org/10. 1007/BFb0066247.

10. Bagh A. Epi/hypo-convergence: the slice topology and saddle point approximations. J. Appl. Anal. 1996;4:13-39. Available from: https://doi.org/10.1515/JAA.1996.13.

11. Soueycatt M. Stabilité quantitative des fonctions convexesconcaves. Topologie épi/hypo-distance, Séminaire d'Anal. Conv, Univ Montpelier II. 1990;20:2.1-2.54.

12. Wright SE. Consistency of primal-dual approximations for convex optimal control problems. SIAM J Control Optim. 1995;33:1489-1509. Available from: https://doi.org/10.1137/ S0363012992240503.

13. Attouch H, Wets RJB. Convergence des points $\mathrm{min} /$ sup et de points fixes. Comp Ren Acad Sci Paris. 1983;296:657-660.

14. Attouch $H$, Azé $D$, Wets RJB. On continuity of the partial Legendre-Fenchel transform: Convergence of sequences of augmented Lagrangian functions, Moreau-Yosida approximates and subdifferential operators, in Fermat-Days 85: Mathematics for Optimization, J.-B. Hiriart-Urruty, ed. North Holland. 1986;p. 1-42. Available from: https://doi.org/10.1016/ S0304-0208(08)72392-2.

15. Attouch H, Azé D, Wets RJB. Convergence of convex-concave saddle functions: continuity properties of the LegendreFenchel transform with applications to convex programming and mechanics. Ann Inst H Poincaré: Anal Nonlinéaire. 1988;5:537-572. Available from: https://doi.org/10.1016/ S0294-1449(16)30335-3.

16. Diem HTH, Khanh PQ. Criteria for epi/hypo convergence of finite-valued bifunctions. Vietnam J Math. 2015;43:439-458. Available from: https://doi.org/10.1007/s10013-015-0139-x.

17. Diem HTH, Khanh PQ. Approximations of optimizationrelated problems in terms of variational convergence. Vietnam J Math. 2016;44:399-417.

18. Jofré A, Wets RJB. Variational convergence of bifunctions: Motivating applications. SIAM J Control Optim. 2014;24:19521977. Available from: https://doi.org/10.1137/120894695.

19. Lopez R. Approximations of equilibrium problems. SIAM J Control Optim. 2012;50:1038-1070. Available from: https:// doi.org/10.1137/110843125.

20. Royset JO, Wets RJB. Lopsided convergence: An extention and its quantification, Math. Program. 2019;177:395-423. Available from: https://doi.org/10.1007/s10107-018-1275-3.

21. Lopez R, Vera C. On the set of weakly efficient minimizers for convex multiobjective programming. Oper Res Lett. 2008;36:651-655. Available from: https://doi.org/10.1016/j. orl.2008.03.002.

22. Rockafellar RT. Minimax theorems and conjugate saddlefunctions. Math Scand. 1964;14:151-173. Available from: https://doi.org/10.7146/math.scand.a-10714.

23. Rockafellar RT. A general correspondence between dual minimax problems and convex programs. Pacific J Math. 1968;25:597-611. Available from: https://doi.org/10.2140/pjm. 1968.25.597.

24. Rockafellar RT. Convex Analysis, Princeton University Press. 1970;Available from: https://doi.org/10.1515/9781400873173.

25. Fenchel W. On conjugate convex functions. Canadian J Math. 1949;1:73-77. Available from: https://doi.org/10.4153/CJM1949-007-x. 
26. Jofré A, Wets RJB. Variational convergence of bivariate functions: Lopsided convergence, Math. Proggram. 2009;116(B):275-295. Available from: https://doi.org/10.1007/ s10107-007-0122-8.
27. Beer G, Rockafellar RT, Wets RJB. A characterization of epiconvergence in terms of convergence of level sets. Proc Amer Math Soc. v;116:753-761. Available from: https://doi.org/10. 1090/S0002-9939-1992-1119262-6. 